\title{
INVENTARIO Y CARACTERIZACIÓN MORFOLÓGICA DE LAGOS Y LAGUNAS DE ALTA MONTAÑA EN LAS PROVINCIAS DE PALENCIA Y LEÓN (ESPAÑA)
}

\section{Inventory and morphological characterization of high mountain lagoons and lakes in the provinces of Palencia and León (Spain)}

\author{
J. F. Fuentes-Pérez*, J. Navarro Hevia, J. Ruiz Legazpi \& A. García-Vega \\ U.D. de Hidráulica e Hidrología. Departamento de Ingeniería Agrícola y Forestal. E.T.S. de Ingenierías Agrarias. \\ Universidad de Valladolid. Avd. Madrid, 57, CP: 34004, Palencia. España. \\ * Autor corresponsal: jfuentes@iaf.uva.es
}

Recibido: 16-03-2015. Aceptado: 06-07-2015. Fecha de publicación on-line: 19/10/2015.

Citation / Cómo citar este artículo: Fuentes-Pérez, J. F., Navarro Hevia, J., Ruiz Legazpi, J. \& García-Vega, A. (2015). Inventario y caracterización morfológica de lagos y lagunas de alta montaña en las provincias de Palencia y León (España). Pirineos, 170, e013. doi: http://dx.doi.org/10.3989/Pirineos.2015.170006

RESUMEN: Los lagos y lagunas de alta montaña no son un rasgo típico del paisaje de la Península Ibérica, sino más bien una excepción en el medio natural, por ello su estudio y conservación presenta gran interés. Este trabajo surge con objeto de inventariar y caracterizar un conjunto de diecisiete complejos lagunares ubicados en las provincias de Palencia y León (Cordillera Cantábrica y Sierra de la Cabrera), sin ningún tipo de restricción por tamaño. Así mismo se describen sus cuencas, ligadas con las masas de agua y que determinarán su futura evolución. Entre otros resultados, cabe señalar que, a pesar de su carácter de cabecera, presentan una red hidrológica compleja y variada, denotando la diversidad de estos sistemas. La composición litológica parece incidir significativamente en la profundidad de las unidades, sin embargo la profundidad y dimensiones de las lagunas dependen en gran medida de las características concretas de su morfogénesis. En general, los lagos y lagunas estudiados parecen ser más frágiles ante posibles alteraciones en sus cuencas al compararlas con otros sistemas de mayor tamaño recogidos en diferentes estudios.

PALABRAS CLAVE: Lagos de alta montaña; cuencas de montaña; inventario; morfometría.

\begin{abstract}
High mountain lakes and lagoons are not a typical feature of the landscape of the Iberian Peninsula, but rather an exception in the natural environment, therefore its study and conservation presents great interest. This study arises in order to inventory and characterize a set of seventeen lagoon complex located over the provinces of Palencia and León, without any kind of size restriction. Likewise their basins are described, since they are related with them and they will determine their future evolution. Among other results, it should be noted that, despite they are situated at high altitude, they have a complex and varied hydrological network, denoting the high diversity of these systems. The lithology factor seems to affect significantly to the depth of the studied units, however depth and dimensions of the lakes are strongly related with the specific characteristics of their morphogenesis. In general, studied lakes and lagoons appear to be more fragile to possible alterations in their catchments if they are compared with bigger systems studied in the specialized literature.
\end{abstract}

KEYWORDS: High mountain lakes; mountain basins; inventory; morphometry. 


\section{Introducción}

En España los lagos y lagunas de alta montaña constituyen una singularidad en el medio natural y, aunque en su mayoría sean pequeños (Toro et al., 2006), tienen gran importancia ya que además de encontrarse en el origen del ciclo hidrológico, son auténticos refugios y reservorios biológicos, excepciones geomorfológicas y sistemas que, en muchas ocasiones, se encuentran inalterados (Toro \& Granados, 2002).

La mayoría de los lagos de alta montaña se originaron por la acción de los hielos glaciares durante el Pleistoceno, por ello podemos considerarlos como ecosistemas jóvenes (Catalan et al., 2006). Este hecho junto con su marcado aislamiento (García-Jurado et al., 2007) han puesto de manifiesto la importancia de su estudio y conservación, ya sea como indicadores del cambio global u otras alteraciones ambientales (Morales-Baquero et al., 1992; Skjelkvåle y Wright, 1998; Battarbee et al., 2002; Catalan et al., 2002; Battarbee, 2005; Catalan et al., 2006), para comprender y observar procesos (Catalan et al., 1992; Toro \& Granados, 2002; Casamitjana et al., 2006; Vega et al., 2005) o simplemente para estudiar la vida en condiciones extremas (Cruz-Pizarro et al., 1994; Almodóvar \& Elvira, 2000; Carrillo et al., 2002; Carrillo et al., 2008; Ordoñez et al., 2009).

Así mismo y dada su excepcionalidad, han surgido grandes trabajos de inventario y caracterización que han incluido muchos de estos sistemas, como El Catálogo de los Lagos Españoles (Pardo, 1948) o El Inventario de Lagos y Humedales de España (Montes, 1995) a nivel nacional o El Catálogo de zonas húmedas de Castilla y León (Decreto 194/1994) y su ampliación (Decreto 125/2001) a nivel de Catilla y León, lo que ha propiciado que tanto la legislación Europea, a través de la Directiva Marco de Agua (Comisión Europea, 2000), como la legislación estatal, mediante las Instrucciones de Planificación Hidrológica Nacional (Orden ARM/2656/2008), intenten asegurar su buen estado químico, hidrológico y ecológico (Arruebo et al., 2009).

No obstante, tanto en el estudio como en el inventario y caracterización de las lagunas de alta montaña, existe un marcado sesgo en cuanto al tamaño se refiere (Fuentes-Pérez, 2009). Esto se debe principalmente a que, por un lado la legislación nacional es una transposición de la europea, donde en general las masas de agua presentan un mayor tamaño, y por otro, a que en muchos casos para establecer redes comparativas es necesario el estudio de masas con características morfológicas y físico-químicas similares (Battarbee et al., 2002), quedando así las de menor tamaño relegadas a un segundo plano. De la misma forma, pequeñas lagunas constituyen en ciertas ocasiones complejos lagunares con masas de mayor entidad (Catalan et al., 2006) (a partir de ahora asociadas) [e.g. Lagunas de Neila (Burgos), Pozo de las Lomas (Palencia), etc.], formando sistemas funcionalmente indivisibles que deben ser estudiados en conjunto. Así mismo, si se considera que históricamente los sistemas con mayor número de estudios han sido los más próximos a los grandes cen- tros universitarios (Pascual et al., 2000) se concluye que muchas de las lagunas peninsulares de menor entidad no han sido inventariadas y mucho menos caracterizadas (Toro et al., 2006). Un caso particular de esta discriminación por tamaño son las masas de la Cordillera Cantábrica donde el $73 \%$ de ellas presenta una superficie inferior a 0,5 ha (Pascual et al., 2000), límite que las suele excluir de cualquier posible inventario.

Por otro lado, teniendo en cuenta que, a pesar de su tamaño, estos lagos y/o lagunas de menor tamaño en ocasiones muestran los mismos procesos morfogenéticos y morfodinámicos que masas mayores (Fuentes-Pérez, 2009), siempre a una escala menor, se pueden considerar en algunos aspectos, como modelos reducidos de las de mayor envergadura, y por lo tanto, surge la oportunidad de obtener con un coste menor (dada sus menores superficies de estudio) conclusiones equiparables o aproximaciones interesantes, haciendo que su inventario y caracterización cobre un gran interés.

Bajo estas premisas y con el fin de solventar, en cierta medida, estas limitaciones surge el presente trabajo. En él se inventarían y caracterizan morfológicamente 17 complejos lagunares, sin ningún tipo de exclusión por tamaño, repartidos en las provincias de Palencia y León (Cordillera Cantábrica y Sierra de la Cabrera). Todo ello con el fin de describir estos sistemas lagunares de alta montaña, que de otra forma no serían objeto de inventario, tener un punto de referencia para poder tomar decisiones futuras y servir como ejemplo de caracterización e iniciativa a considerar.

\section{2. Área de Estudio}

Los lagos y lagunas considerados forman parte de la selección realizada para el proyecto del Plan Nacional REN 2003-03718/HD, "Bases científicas para la definición del estado ecológico de lagos y lagunas de montaña de la cuenca del Duero". La mayor parte de estas masas de agua se sitúan en el norte de las provincias de Palencia y León, en la Cordillera Cantábrica, y el resto al sur de la provincia de León, en la Sierra de la Cabrera (Figura 1), todas ellas pertenecientes a las cuencas del río Duero y del río Miño.

En la Tabla 1 se definen las coordenadas geográficas UTM de cada una de ellas así como las principales composiciones litológicas de sus cuencas, de acuerdo a los mapas geológicos nacionales del Instituto Geológico y Minero de España (IGME) a escala 1:50000. Teniendo en cuenta su situación y litología, los lagos y lagunas estudiados pueden dividirse en tres grupos o regiones (Figura 1). El grupo I se corresponde con las masas más orientales. Éstas se encuentran en una zona compuesta principalmente por pizarras, conglomerados y calizas, si bien cabe resaltar que el Pozo de las Lomas (PA1) se sitúa sobre un stock granítico que toma el nombre de la cumbre a la que da origen, Peña Prieta (2539 m.s.n.m.) y Fuentes Carrionas y Hoyo Empedrado (PA2 y LE1) sobre la formación Lechada de lutitas y areniscas westfalienses, ambas formaciones ampliamente estudiadas por Gallastegui et al. (1990). El grupo II, situado al noroeste, se encuentra 


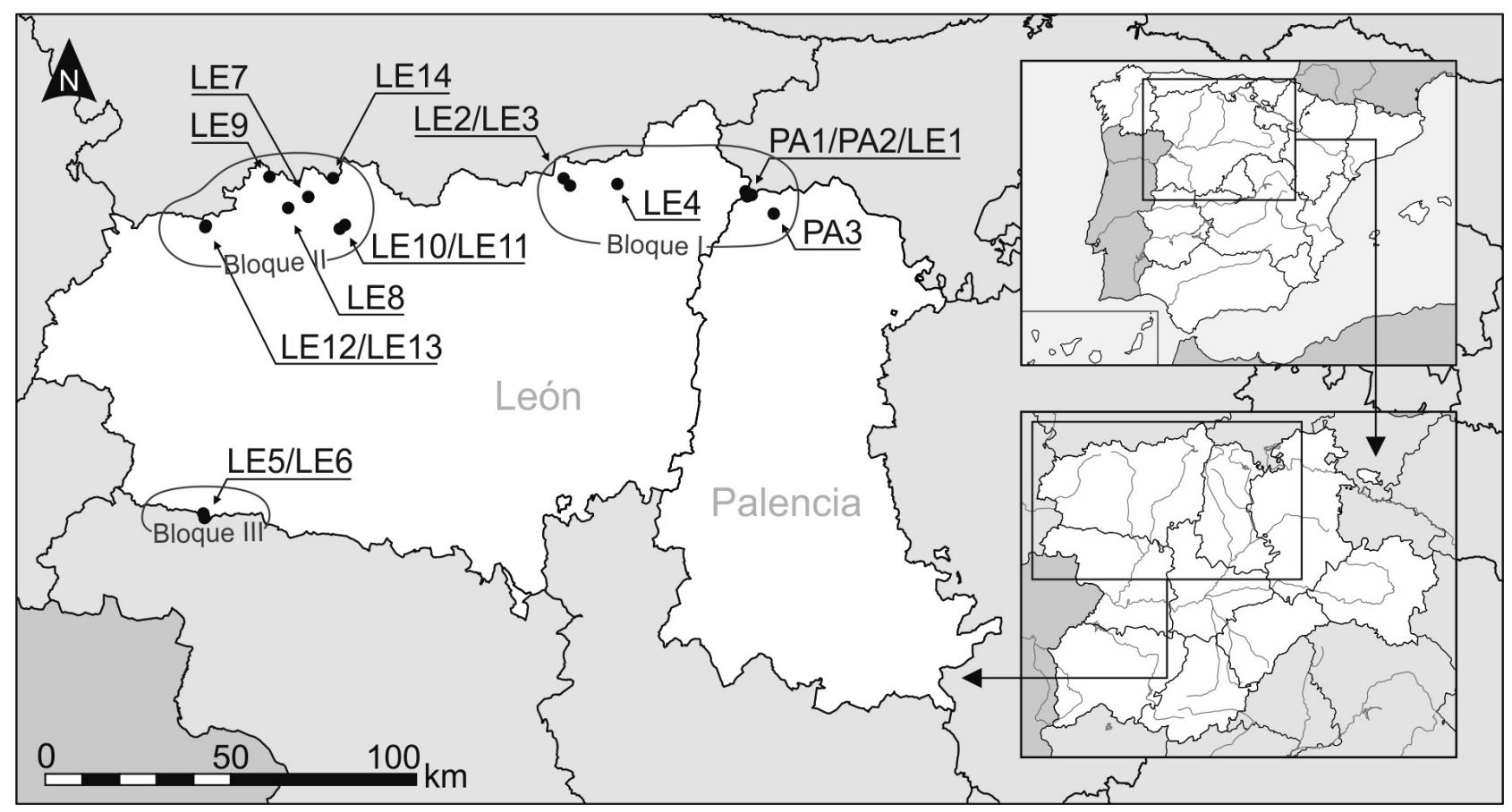

Figura 1: Situación de los complejos lagunares estudiados.

Figure 1: Situation of the studied lakes.

sobre una región de pizarras y calizas excepto por las lagunas El Miro y Pozo Cheiroso (LE12 y LE13) que se asientan sobre cuarcitas areniscas y pizarras correspondientes a serie de los Cabos y la laguna El Castro (LE8) que lo hace sobre areniscas y pizarras de la cuenca Estefaniense de Villablino. Por último el grupo III, en el suroeste, se encuentra formado por dos lagos próximos de diferente cuenca (Truchillas y Malicioso) que se hallan asentados sobre cuarcitas blancas.

Todas las masas de agua estudiadas, dada su ubicación, se encuentran al amparo de diferentes figuras de protección (Tabla 1).

\section{Materiales y métodos}

Con objeto de localizar y delimitar las masas de agua objeto de estudio, en una primera fase, fueron situadas sobre los mapas topográficos del Instituto Geográfico Nacional (IGN) a escala 1:25000 y ortofotografías aéreas. Con esta información, siempre que no existieran, se trazaban las mejores rutas de acceso. Así mismo, en un intento de caracterización previa se analizaron los diferentes inventarios existentes, donde únicamente se localizaron las de mayor envergadura. En este sentido, cabe señalar que algunos de estos trabajos fueron realizados mediante mapas topográficos con escalas espaciales grandes [e.g. la escala de análisis utilizada por Pardo (1948) en su investigación fue de 1:50000, necesaria debido a la envergadura de dicho trabajo], lo que conllevó a ciertas desviaciones en la estimación previa de la superficie y la forma de algunas lagunas.
Posteriormente, por un lado se realizó una caracterización in situ de cada unidad del complejo seleccionado, y por otro lado, en gabinete se estudiaron las cuencas vertientes de cada una mediante los mapas vectorizados del IGN a escala 1:25000. Este estudio multiescalar era necesario dado que, de forma general, existe una estrecha relación entre las masas de agua y las características de sus cuencas (Catalan et al., 2006), que no siempre es obvia dada la cantidad de variables implicadas (Håkanson, 2005).

\subsection{Caracterización de las masas}

Los trabajos de campo tuvieron lugar durante el verano y otoño de 2008 (desde el 14/07/08 hasta el 15/10/08). Se diseñó una ficha de caracterización que recogía diferentes aspectos de cada laguna (posibles procesos morfogenéticos, origen hidrológico, forma, conectividad, etc.), que era posteriormente completada, evaluada y contrastada en gabinete (Anexo I). Así mismo, se llevó a cabo el levantamiento topográfico de la lámina de agua de cada unidad mediante GPS (Garmin eTrex Vista HCx) y la batimetría del transecto de mayor longitud en intervalos de 1 metro mediante un profundímetro (HONDEX PS-7).

Estas lagunas en numerosas ocasiones forman complejas redes hidrológicas, ya sean con arroyos o masas de agua asociadas, con un alto grado de conectividad y dependencia. Estas redes se definían mediante recorridos perimetrales, utilizando GPS, y analizando la topografía y los flujos de escorrentía. 


\section{4 • J. F. FUENTES-PÉREZ, J. NAVARRO HEVIA, J. RUIZ LEGAZPI, A. GARCÍA-VEGA}

Tabla 1: Situación de los lagos y lagunas estudiadas, litología y figuras de protección.

Table 1: Situation of the studied lakes, lithology and protection categories.

\begin{tabular}{|c|c|c|c|c|c|c|}
\hline \multirow[b]{2}{*}{ Código } & \multirow[b]{2}{*}{ Nombre } & \multicolumn{3}{|c|}{ Situación (Datum ETRS89) } & \multirow[b]{2}{*}{ Litología } & \multirow[b]{2}{*}{ Régimen de Protección } \\
\hline & & Municipio & $\begin{array}{l}\text { UTM } \\
(\mathrm{km})\end{array}$ & \begin{tabular}{|c|} 
Altitud \\
$(\mathrm{m})$
\end{tabular} & & \\
\hline PA1.1 & Pozo de las Lomas & \multirow{5}{*}{$\begin{array}{l}\text { Velilla de } \\
\text { Río Carrión }\end{array}$} & $30 \mathrm{~T} 3584763$ & 2055 & \multirow{5}{*}{$\begin{array}{l}\text { Granodiorita/tonalita (Stock Peña Prieta) } \\
\text { (Gallastegui et al., 1990) }\end{array}$} & \multirow{7}{*}{$\begin{array}{l}\text { Parque Natural, Catálogo } \\
\text { Regional de Zonas } \\
\text { Húmedas de Interés } \\
\text { Especial, ZEPA y LIC }\end{array}$} \\
\hline PA1.2 & Asociada & & 30T3584764 & 2129 & & \\
\hline PA1.3 & Asociada & & 30T3584764 & 2129 & & \\
\hline PA1.4 & Asociada & & 30T3584764 & 2129 & & \\
\hline PA1.5 & Asociada & & 30T3584764 & 2129 & & \\
\hline PA2.1 & Fuentes Carrionas & \multirow[b]{3}{*}{$\begin{array}{l}\text { Cervera } \\
\text { de Pisuerga }\end{array}$} & 30T3594764 & 2200 & \multirow{2}{*}{$\begin{array}{l}\text { Alternancia arenoso-pelítica con intercalación de niveles de conglo- } \\
\text { merados predominantemente silíceos y brechas calcáreas y calizas } \\
\text { (formación Lechada) (Gallastegui et al., 1990) }\end{array}$} & \\
\hline PA2.2 & Asociada & & 30T3594764 & 2195 & & \\
\hline PA3 & Pozo Oscuro & & $30 \mathrm{~T} 3654759$ & 2024 & $\begin{array}{l}\text { Cuarcitas estratificadas (formación Murcia) (Rodríguez } \\
\text { Fernández, 1985) }\end{array}$ & $\begin{array}{l}\text { Parque Natural y } \\
\text { Catálogo Regional de } \\
\text { Zonas Húmedas de } \\
\text { Interés Especial } \\
\end{array}$ \\
\hline LE1 & Hoyo Empedrado & $\begin{array}{l}\text { Boca de } \\
\text { Huérgano }\end{array}$ & $30 \mathrm{~T} 3574765$ & 2073 & $\begin{array}{l}\text { Alternancia arenoso-pelítica con intercalación de niveles de } \\
\text { conglomerados predominantemente silíceos y brechas calcáreas } \\
\text { y calizas (formación Lechada) (Gallastegui et al., 1990) }\end{array}$ & \multirow{2}{*}{$\begin{array}{l}\text { Parque Regional, ZEPA } \\
\text { y LIC }\end{array}$} \\
\hline LE2 & Robledo & \multirow[b]{2}{*}{$\begin{array}{l}\text { Puebla } \\
\text { de Lillo }\end{array}$} & 30T3104766 & 1435 & $\begin{array}{l}\text { Calizas grises oscuras biomicríticas (formación Barcaliente) } \\
\text { y pizarras grises o negras con niveles de areniscas (formación } \\
\text { Fresnedo) (Rodríguez Fernández, 1990a) }\end{array}$ & \\
\hline LE3 & Ausente & & 30T3084768 & 1740 & Cuarzitas (formación Barrios) (Rodríguez Fernández, 1990a) & $\begin{array}{l}\text { Parque Regional, } \\
\text { Catalogo Regional de } \\
\text { Zonas Húmedas de } \\
\text { Interés Especial, ZEPA } \\
\text { y LIC }\end{array}$ \\
\hline LE4 & Mampodre & Maraña & $30 \mathrm{~T} 3234767$ & 1445 & $\begin{array}{l}\text { Calizas negras (formación Barcaliente), calizas rojas y radiolitas } \\
\text { (formación Alba), pizarras y liditas negras (formación Vega- } \\
\text { mián), microconglomerados silícios, areniscas y calizas (forma- } \\
\text { ción Emita) (Rodríguez Fernández, 1990b) }\end{array}$ & $\begin{array}{l}\text { Parque Regional, ZEPA } \\
\text { y LIC }\end{array}$ \\
\hline LE5 & Truchillas & \multirow{2}{*}{ Truchas } & 29T7074674 & 1755 & \multirow{2}{*}{ Cuarcitas blancas (Rodríguez Fernández, 1982b) } & \multirow{2}{*}{$\begin{array}{l}\text { Monumento natural, } \\
\text { ZEPA y LIC }\end{array}$} \\
\hline LE6 & Malicioso & & $29 \mathrm{~T} 7074675$ & 1855 & & \\
\hline LE7 & De Babia & Cabrillanes & 29T7294762 & 1435 & $\begin{array}{l}\text { Dolomías, calizas y pizarras (grupo Vid) (Rodríguez Fernández, } \\
\text { 1982a) }\end{array}$ & $\begin{array}{l}\text { Parque Natural, Catálogo } \\
\text { Regional de Zonas } \\
\text { Húmedas de Interés } \\
\text { Especial, Reserva de la } \\
\text { Biosfera, ZEPA y LIC } \\
\end{array}$ \\
\hline LE8 & El Castro & \multirow[b]{2}{*}{ Villablino } & $29 \mathrm{~T} 7244759$ & 1305 & $\begin{array}{l}\text { Areniscas, pizarras y niveles de Carbón (Rodríguez Fernández, } \\
\text { 1982a) }\end{array}$ & \multirow{2}{*}{$\begin{array}{l}\text { Espacio Natural, Reserva } \\
\text { de la Biosfera, ZEPA } \\
\text { y LIC }\end{array}$} \\
\hline LE9 & La Chagunona & & 29T7184767 & 1873 & $\begin{array}{l}\text { Areniscas, areniscas feldespáticas, cuarcitas, pizarras y conglo- } \\
\text { merados (formación Herrería o Candana) (Rodríguez Fernández, } \\
\text { 1982a) }\end{array}$ & \\
\hline LE10 & Chao & \multirow{2}{*}{$\begin{array}{l}\text { San } \\
\text { Emiliano }\end{array}$} & $29 \mathrm{~T} 7394755$ & 1820 & \begin{tabular}{|l|} 
Pizarras negras ampelíticas con intercalaciones de areniscas \\
(formación Formigosa) (Rodríguez Fernández, 1998)
\end{tabular} & \multirow{2}{*}{$\begin{array}{l}\text { Parque Natural, ZEPA } \\
\text { y LIC }\end{array}$} \\
\hline LE11 & Bustagil & & $29 \mathrm{~T} 7384754$ & 1734 & $\begin{array}{l}\text { Pizarras verdes, lutitas grises y areniscas (formación Oville) } \\
\text { (Rodríguez Fernández, 1998) }\end{array}$ & \\
\hline LE12 & El Miro & \multirow[b]{2}{*}{$\begin{array}{l}\text { Palacios } \\
\text { del Sil }\end{array}$} & 29T7014753 & 1785 & \multirow[b]{2}{*}{$\begin{array}{l}\text { Cuarcitas, areniscas y pizarras (serie de Los Cabos) (Huerga } \\
\text { Rodríguez, 1981) }\end{array}$} & \multirow{2}{*}{$\begin{array}{l}\text { Espacio Natural, Reserva } \\
\text { de la Biosfera, Catálogo } \\
\text { Regional de Zonas } \\
\text { Húmedas de Interés } \\
\text { Especial, ZEPA y LIC } \\
\end{array}$} \\
\hline LE13 & Pozo Cheiroso & & 29T7024752 & 1790 & & \\
\hline LE14 & Congosto & \begin{tabular}{|l|} 
San \\
Emiliano
\end{tabular} & 29T7354768 & 1955 & $\begin{array}{l}\text { Pizarras y areniscas (serie Huergas) y calizas (serie Portilla) } \\
\text { (Rodríguez Fernández, 1982c) }\end{array}$ & $\begin{array}{l}\text { Parque Natural, ZEPA } \\
\text { y LIC }\end{array}$ \\
\hline
\end{tabular}


Posteriormente los datos fueron digitalizados mediante el programa AutoCAD. Por un lado el transecto, obteniendo el perfil batimétrico para estimar las diferentes profundidades, y por otro lado, la superficie de la lámina, superponiéndola tanto a la ortofoto como a la cartografía digitalizada para contrastar posibles incoherencias.

Mediante el procesado de los datos obtenidos en los trabajos de campo se obtuvieron los diferentes parámetros morfométricos para cada una de las unidades estudiadas: perímetro $\left(\mathrm{P}_{\mathrm{L}}\right)$, superficie $\left(\mathrm{S}_{\mathrm{L}}\right)$, longitud $\left(\mathrm{L}_{\max }\right)$, anchuras $\left(\mathrm{B}_{\max }\right)$, profundidades máxima $\left(\mathrm{Z}_{\max }\right)$ y media $\left(\mathrm{Z}_{\text {med }}\right)$, índices de forma $\left(\mathrm{C}_{\mathrm{G}}\right)$ y otros derivados como el volumen $\left(\mathrm{V}_{\mathrm{L}}\right)$, estimado como $\mathrm{S}_{\mathrm{L}} \cdot \mathrm{Z}_{\text {med }}$.

El origen de cada laguna, así como los posibles modelados periglaciares, se determinaron mediante la observación in situ, el estudio de mapas topográficos y la revisión de bibliografía.

Una vez definidas todas las variables se utilizó, por un lado, el análisis de la varianza (ANOVA) para determinar si las relaciones entre estas variables eran estadísticamente significativas en relación con la litología $\mathrm{y}$, por otro lado, el análisis de regresión para determinar la relación entre las diferentes variables continuas estudiadas.

\subsection{Caracterización de las cuencas vertientes}

Para comprender el funcionamiento de cada una de las lagunas así como para confirmar el origen de las mismas, es necesario realizar un estudio a mayor escala. En este sentido, se llevó a cabo el análisis de cada una de las cuencas de los complejos estudiados mediante las hojas vectorizadas de los mapas topográficos del IGN a escala 1:25000. Entre otros parámetros de cuenca se calcularon la superficie $\left(\mathrm{S}_{\mathrm{C}}\right)$, el índice de forma $\left(\mathrm{C}_{\mathrm{G}}\right)$, la curva hipsométrica (representación gráfica de la superficie acumulada frente a la cota) y la pendiente media (I), de acuerdo a Martínez de Azagra y Navarro (1996).

Así mismo con objeto de comparar la vulnerabilidad de los complejos lagunares estudiados con otros sistemas peninsulares se calculó el cociente entre el volumen de las masas de agua (estimado como $\mathrm{Z}_{\text {med }} \cdot \mathrm{S}_{\mathrm{L}}$ ) y la superficie de la cuenca $\left(\mathrm{S}_{\mathrm{C}}\right)$. Se trata de un índice que ayuda a interpretar el grado de vulnerabilidad de las masas ante actuaciones en su cuenca vertiente (Catalan, 1987; Vega et al., 2005).

\section{Resultados y discusión}

Los lagos y lagunas estudiadas tienen un tamaño pequeño al compararlas con las descritas en otros sistemas montañosos de la Península Ibérica, como pueden ser los Pirineos (Catalan et al., 1992) o el Sistema Central (Toro et al., 2006). Este menor tamaño determina una mayor influencia de las condiciones ambientales externas. De forma general, se trata de unidades pequeñas donde más del $50 \%$ presentan superficies inferiores a 0,5 ha. Esto se ajusta a la realidad peninsular donde el $75 \%$ de las lagunas presenta una superficie inferior a media hectárea
(Toro et al., 2006). Así mismo, el total de las masas de agua estudiadas ocupan una superficie de 220 ha, destacando que el $67,15 \%$ de esta superficie corresponde tan solo a 4 láminas de agua [lago de Babia (LE7), lago de Truchillas (LE5), lago del Ausente (LE3) y lago de Fuentes Carrionas (PA2)] (Figura 2). Gran parte de las masas de agua de mayor tamaño pertenecen al primer y tercer grupo, correspondiendo con las lagunas de litología más dura. A pesar de que El lago de Babia pertenece al grupo II, debe su mayor superficie a su origen por una transfluencia glaciar del río Sil (Santos González, 2012). Así mismo, predominan las lagunas con forma redonda y ovalada (Tabla 3 y Figuras 3 y 4).

Los 17 complejos lagunares se distribuyen en un amplio rango altitudinal, que presenta como cota superior los 2200 m.s.n.m. y como inferior los 1305 m.s.n.m. Más del $80 \%$ de estas lagunas aparecen ubicadas a una altitud superior a los 1500 m.s.n.m., límite considerado como frontera para las condiciones de alta montaña (Pascual et al., 2000).

La mayoría de los lagos y lagunas estudiados deben su origen a un circo glaciar, aunque también las hay de origen yuxtaglaciar y por transfluencia glaciar (Tabla 2). El origen es menos apreciable a medida que se desciende en altitud dada la mayor intensidad de los procesos sedimentarios (deposición y acumulación) y las alteraciones antrópicas (Battarbee, 2005). La única masa de agua que parece tener un sistema morfogenético distinto es la laguna Chagunona, cuyo origen se debe a un factor combinado entre el glaciarismo y procesos kársticos.

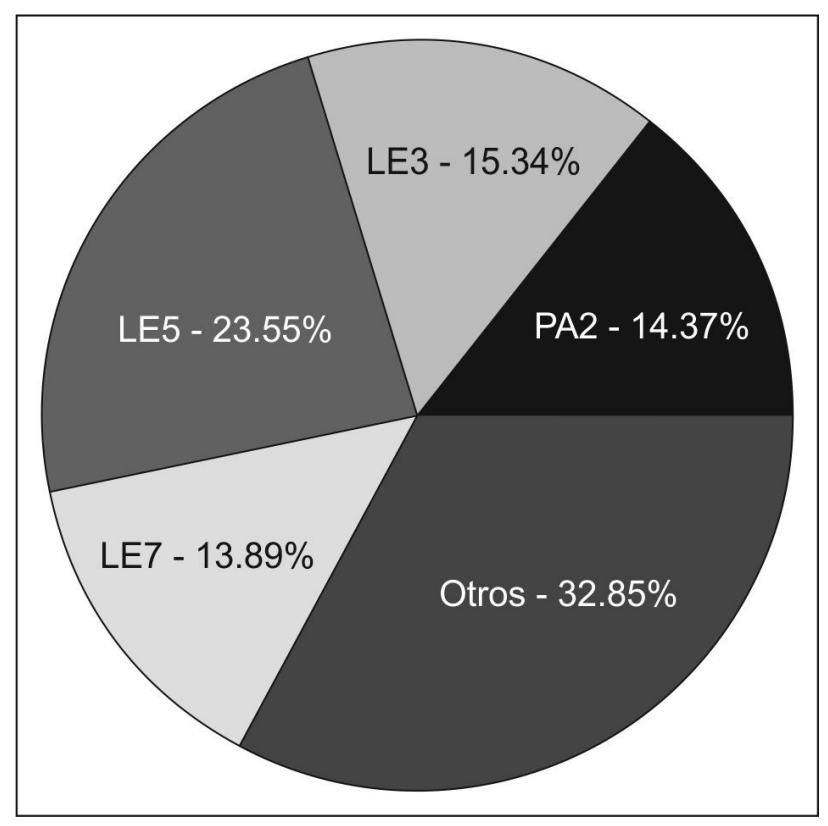

Figura 2: Distribución de la superficie de las unidades estudiadas. PA2: Fuentes Carrionas; LE3: Ausente; LE5: Truchillas; LE7: Babia.

Figure 2: Distribution of surface in the studied lakes. PA2: Fuentes Carrionas; LE3: Ausente; LE5: Truchillas; LE7: Babia. 
Tabla 2: Origen de las unidades lagunares estudiadas.

Table 2: Origin of the studied lakes.

\begin{tabular}{|c|c|c|c|}
\hline Código & Nombre & Origen & Observaciones \\
\hline PA1 & $\begin{array}{l}\text { Pozo de las } \\
\text { Lomas }\end{array}$ & Circo glaciar & $\begin{array}{l}\text { Circo glaciar coronado por las Agujas de Cardaño con dos cubetas de sobre-excavación, estando la inferior ocupada } \\
\text { por el Pozo de las Lomas. }\end{array}$ \\
\hline PA2 & $\begin{array}{l}\text { Fuentes } \\
\text { Carrionas }\end{array}$ & Circo glaciar & $\begin{array}{l}\text { El circo de Fuentes Carrionas posee un complejo morrénico frontal (formado por cuatro morrenas frontales) que } \\
\text { cierra el Pozo de Fuentes Carrionas y su asociada. }\end{array}$ \\
\hline PA3 & Pozo Oscuro & Circo glaciar & $\begin{array}{l}\text { El arco morrénico que define el margen de la vertiente noreste indica un posible origen por glaciar de circo. Ade- } \\
\text { más, su cuenca presenta también forma de circo, rodeada por escarpes rocosos con numerosas cárcavas. }\end{array}$ \\
\hline LE1 & $\begin{array}{l}\text { Hoyo } \\
\text { Empedrado }\end{array}$ & Circo glaciar & $\begin{array}{l}\text { Esta laguna se halla situada en el denominado circo de Hoyo Empedrado, protegido y alimentado por las paredes } \\
\text { granodioríticas de las Agujas de Cardaño. }\end{array}$ \\
\hline LE2 & Robledo & Circo glaciar & $\begin{array}{l}\text { El valle que sucede a la laguna (Valle de Iyarga) presenta extensos depósitos glaciares (Rodríguez Fernández, } \\
\text { 1990a) y teniendo en cuenta la propia morfología de la cuenca de esta laguna (Fig. 3), parece factible que durante } \\
\text { la fase glaciar se produjera en esta cuenca una acumulación de nieve (un glaciar de circo de poca magnitud). Esta } \\
\text { acumulación, debido al estrechamiento final de la cuenca, ofrecía mayor resistencia donde en la actualidad se asien- } \\
\text { ta la laguna, produciendo una sobreexcavación. }\end{array}$ \\
\hline LE3 & Ausente & Circo glaciar & $\begin{array}{l}\text { Se sitúa en una cubeta sobreexcavada por el circo glaciar del Ausente. Los indicios de esta actividad perduran a día } \\
\text { de hoy como formas heredadas, tales como el escarpe rocoso en forma de circo que antecede a la laguna o el arco } \\
\text { morrénico que le precede. }\end{array}$ \\
\hline LE4 & Mampodre & Circo glaciar & $\begin{array}{l}\text { Esta laguna se halla situada entre los depósitos glaciales del denominado circo Mampodre. Existen abundantes tills } \\
\text { (sobre todo cerca de la localidad Maraña) de los que algunos presentan forma de arco, o la cabecera de la cuenca, } \\
\text { conformada por tres circos unidos en su base. }\end{array}$ \\
\hline LE5 & Truchillas & Circo glaciar & $\begin{array}{l}\text { El lago ocupa una depresión que fue sobreexcavada por un glaciar de circo (presenta una corona de roca cuarcítica } \\
\text { y dos morrenas laterales). El valle que sucede al lago presenta forma de artesa hepicilíndrica, modelado ahora por el } \\
\text { arroyo del lago y los bloques erráticos que arrancó la lengua de hielo a su paso, que ahora aparecen desperdigados } \\
\text { por toda la zona que sucede a la laguna. }\end{array}$ \\
\hline LE6 & Malicioso & Circo glaciar & $\begin{array}{l}\text { La cabecera del valle del reguero del Malicioso presenta un doble circo, siendo uno de ellos el correspondiente a } \\
\text { la cubeta donde se asienta el lago Malicioso. Dicha cubeta se conformó por una sobreexcavación profunda (lo que } \\
\text { explicaría la gran profundidad del lago) delimitada en la actualidad por el circo cuarcítico que antecede a la masa y } \\
\text { los derrubios en forma de arco que la suceden. De la misma forma se pueden apreciar por todo el valle que sucede } \\
\text { al lago grandes bloques erráticos. }\end{array}$ \\
\hline LE7 & De Babia & $\begin{array}{l}\text { Transfluencia } \\
\text { glaciar del } \\
\text { río Sil }\end{array}$ & $\begin{array}{l}\text { La ausencia de una cabecera en el valle se explica por las grandes dimensiones que alcanzó el glaciar del Sil, } \\
\text { haciendo que una parte de esta gran lengua difluyera a través del collado de Babia, sobre-excavando la cubeta que } \\
\text { ocupa el lago y depositando arcos morrénicos y bloques erráticos (Santos González \& Fernández Martínez, 2011). }\end{array}$ \\
\hline LE8 & El Castro & Yuxtaglaciar & $\begin{array}{l}\text { La lengua glaciar que la originó se situó por detrás de los abanicos aluviales proglaciares de Villablino. Su forma- } \\
\text { ción se produjo por la deglaciación lenta en toda la zona alternada con pequeños avances del frente glaciar que no } \\
\text { sobrepasaron el límite máximo inicial (Leira et al., 1997). }\end{array}$ \\
\hline LE9 & La Chagunona & Mixto & $\begin{array}{l}\text { Origen debido a un factor combinado entre el glaciarismo (la actividad glaciar de la zona queda definida por los } \\
\text { depósitos glaciares y la forma de artesa hepicilíndrica del valle de Sosas, pareciendo razonable pensar que la } \\
\text { orografía propició una zona de acumulación de nieve, acompañada de su sobreexcavación) y procesos kársticos } \\
\text { (formación de la dolina). }\end{array}$ \\
\hline LE10 & Chao & Circo Glaciar & $\begin{array}{l}\text { La morfología glaciar en la cabecera del arroyo de Riolago está constituida por circos glaciares, con cubetas de } \\
\text { sobreexcavación y cierres morrénicos que alojan las lagunas del Lago y del Chao (Plan hidrológico de la parte } \\
\text { española de la demarcación hidrográfica del Duero. Anejo 3: zonas protegidas, 2012). }\end{array}$ \\
\hline LE11 & Bustagil & Circo glaciar & $\begin{array}{l}\text { Existe una cresta cuarzoarenítica que corona el oeste de la laguna (que responde a la forma propia heredada por la } \\
\text { presencia de un glaciar de circo) y extensos depósitos glaciares desde la propia laguna hasta aguas abajo del arroyo } \\
\text { Bustagil. } \\
\text { El valle de Bustagil se encuentra en la vertiente norte de la sierra de Villabandín. Dicha sierra está formada por nu- } \\
\text { merosas redes de valles en artesa con nítidos circos en sus cabeceras (Frochoso Sánchez \& Castañón Álvarez, 1997). }\end{array}$ \\
\hline LE12 & El Miro & Yuxtaglaciar & $\begin{array}{l}\text { La lengua glaciar que la originó se situó por detrás de los abanicos aluviales proglaciares de Villablino. Su forma- } \\
\text { ción se produjo por la deglaciación lenta en toda la zona alternada con pequeños avances del frente glaciar que no } \\
\text { sobrepasaron el límite máximo inicial (Leira et al., 1997). }\end{array}$ \\
\hline LE13 & Pozo Cheiroso & Circo glaciar & $\begin{array}{l}\text { Se presenta un circo sobreexcavado en roca cuarcítica que corona a la laguna, al que le sigue un valle en artesa } \\
\text { hepicicloidal. }\end{array}$ \\
\hline LE14 & Congosto & Circo glaciar & $\begin{array}{l}\text { La cabecera de la cuenca tiene forma de circo, limitada por las crestas calizas situadas en el occidente. Se presenta } \\
\text { también un arco morrénico consolidado en el oriente. }\end{array}$ \\
\hline
\end{tabular}


Tabla 3: Características físicas de las unidades lagunares estudiadas.

Table 3: Physical characteristics of the studied lakes.

\begin{tabular}{|c|c|c|c|c|c|c|c|c|c|c|}
\hline Código & $S_{L}\left(m^{2}\right)$ & $P_{L}(m)$ & $\mathrm{L}_{\max }(\mathrm{m})$ & $B_{\text {max }}(m)$ & $\mathrm{Z}_{\text {max }}(\mathrm{m})$ & $\mathrm{Z}_{\text {med }}(\mathrm{m})$ & \multicolumn{2}{|c|}{ Tipología $\left(\mathrm{C}_{\mathrm{G}}\right)$} & Conectividad $^{1}$ & Origina $^{3}$ \\
\hline PA1.1 & 19832.48 & 607.04 & 198.43 & 145.14 & 8.20 & 6.16 & 1.21 & Redonda & Exorreica & Arroyo de las Lomas \\
\hline PA1.2 & 130.79 & 58.90 & 16.27 & 14.51 & $<0.50$ & $<0.50$ & 1.44 & Ovalada & Exorreica & Asociada 1.2 \\
\hline PA1.3 & 611.18 & 129.38 & 47.81 & 21.56 & 1.30 & 0.87 & 1.47 & Ovalada & Exorreica & Asociada 2.1 \\
\hline PA1.4 & 116.95 & 48.34 & 16.52 & 12.29 & $<0.50$ & $<0.50$ & 1.25 & Redonda & Exorreica & Asociada 2.2 \\
\hline PA1.5 & 1968.09 & 219.09 & 67.03 & 46.55 & 1.60 & 1.35 & 1.38 & Ovalada & Exorreica & Las Lomas \\
\hline PA2.1 & 30342.42 & 710.82 & 260.68 & 159.92 & 11.70 & 6.47 & 1.14 & Redonda & Exorreica & Asociada 1 \\
\hline PA2.2 & 2193.58 & 214.87 & 76.60 & 38.78 & 3.50 & 2.34 & 1.28 & Ovalada & Exorreica & Río Carrión \\
\hline PA3 & 2162.77 & 183.80 & 73.24 & 38.85 & 6.70 & 3.62 & 1.11 & Redonda & Endorreica & - \\
\hline LE1 & 1183.09 & 161.73 & 53.08 & 33.39 & $<0.50$ & $<0.50$ & 1.32 & Ovalada & Exorreica & Arroyo de Hoyo Empedrado \\
\hline LE2 & 12153.74 & 481.19 & 159.20 & 121.94 & 0.00 & 0.00 & 1.22 & Redonda & Endorreica parcial $^{2}$ & - \\
\hline LE3 & 34730.43 & 715.19 & 249.45 & 211.82 & 11.80 & 6.38 & 1.07 & Redonda & Endorreica & - \\
\hline LE4 & 914.12 & 143.14 & 47.26 & 27.58 & $<0.50$ & $<0.50$ & 1.33 & Ovalada & Exorreica & Reguero Mampodre \\
\hline LE5 & 53327.95 & 1083.74 & 431.32 & 181.70 & 5.60 & 3.28 & 1.31 & Ovalada & Exorreica & Arroyo del Lago \\
\hline LE6 & 5756.93 & 305.03 & 115.63 & 72.66 & 8.80 & 5.63 & 1.13 & Redonda & Exorreica & Reguero del Malicioso \\
\hline LE7 & 31452.14 & 925.98 & 407.76 & 125.78 & $<2.00$ & $<2.00$ & 1.46 & Ovalada & Endorreica parcial $^{2}$ & Arroyo de Lago \\
\hline LE8 & 7800.77 & 390.93 & 126.25 & 109.74 & $<0.50$ & $<0.50$ & 1.24 & Redonda & Endorreica & - \\
\hline LE9 & 4357.10 & 274.23 & 105.42 & 63.41 & $<0.50$ & $<0.50$ & 1.16 & Redonda & Endorreica parcial & Río de Sosas \\
\hline LE10 & 6505.14 & 505.09 & 206.51 & 65.68 & 0.00 & 0.00 & 1.75 & Fusiforme & Exorreica & Arroyo de la Señal \\
\hline LE11 & 1385.16 & 153.49 & 52.81 & 39.77 & 0.00 & 0.00 & 1.15 & Redonda & Endorreica parcial $^{2}$ & Arroyo de Bustagil \\
\hline LE12 & 810.49 & 110.87 & 41.20 & 26.76 & $<0.50$ & $<0.50$ & 1.09 & Redonda & Endorreica & - \\
\hline LE13 & 5231.30 & 299.68 & 109.01 & 66.77 & 5.70 & 4.39 & 1.16 & Redonda & Endorreica parcial $^{2}$ & Arroyo la Braña el Monte \\
\hline LE14 & 3467.68 & 271.75 & 78.36 & 63.10 & $<0.50$ & $<0.50$ & 1.29 & Ovalada & Endorreica parcial $^{2}$ & Río de la Majúa \\
\hline
\end{tabular}

${ }^{1}$ Refleja la situación observada en campo.

${ }^{2}$ Endorreica en el momento de estudio pero con constancia de exorreísmo.

${ }^{3}$ Unidad a la que da origen el lago o la laguna.

Simbología

\begin{tabular}{|c|c|l|}
\hline Símbolo & Unidad & \multicolumn{1}{|c|}{ Significado } \\
\hline $\mathrm{S}_{\mathrm{L}}$ & $\mathrm{m}^{2}$ & Superficie del lago o laguna \\
\hline $\mathrm{P}_{\mathrm{L}}$ & $\mathrm{m}$ & Perímetro del lago o laguna \\
\hline $\mathrm{L}_{\max }$ & $\mathrm{m}$ & Longitud máxima del lago o laguna \\
\hline $\mathrm{B}_{\max }$ & $\mathrm{m}$ & Anchura máxima perpendicular a la longitud máxima del lago o laguna \\
\hline $\mathrm{Z}_{\max }$ & $\mathrm{m}$ & Profundidad máxima del lago o laguna \\
\hline $\mathrm{Z}_{\text {med }}$ & $\mathrm{m}$ & Profundidad media del lago o laguna \\
\hline $\mathrm{C}_{\mathrm{C}}$ & - & Coeficiente o índice de forma de Gravelius \\
\hline $\mathrm{S}_{\mathrm{C}}$ & $\mathrm{m}^{2}$ & Superficie de la cuenca \\
\hline $\mathrm{P}_{\mathrm{C}}$ & $\mathrm{m}$ & Perímetro de la cuenca \\
\hline $\mathrm{L}_{\mathrm{C}}$ & $\mathrm{m}$ & Longitud de la cuenca \\
\hline $\mathrm{H}_{\max }$ & $\mathrm{m}$ & Altitud máxima de la cuenca \\
\hline $\mathrm{H}_{\operatorname{med}}$ & $\mathrm{m}$ & Altitud media de la cuenca \\
\hline $\mathrm{H}_{\min }$ & $\mathrm{m}$ & Altitud mínima de la cuenca \\
\hline $\mathrm{I}$ & $\%$ & Pendiente media de la cuenca \\
\hline $\mathrm{V}_{\mathrm{L}}$ & $\mathrm{m}^{3}$ & Volumen del lago o laguna $\left(\mathrm{Z}_{\text {med }} \cdot \mathrm{S}\right.$ L \\
\hline $\mathrm{A}_{\operatorname{med}}$ & $\mathrm{m}$ & Altura media de la cuenca \\
\hline
\end{tabular}




\section{8 • J. F. FUENTES-PÉREZ, J. NAVARRO HEVIA, J. RUIZ LEGAZPI, A. GARCÍA-VEGA}

Existe una gran diversidad muestral en cuanto a la profundidad se refiere, si bien son coherentes con sus tamaños, las características litológicas y su morfogénesis (Tablas 1 y 2). De forma general, la profundidad de las lagunas es mayor a medida que aumentan en superficie $\left(p\right.$-valor $=0.008$ para $Z_{\max }$ y $p$-valor $=0.012$ para $Z_{\text {med }}$ ), lo que también se observa en las lagunas de alta montaña del Pirineo (Catalan et al., 1992).

La relación entre la profundidad y litología es estadísticamente significativa [en general, los lagos de mayor profundidad media y máxima se sitúan en los grupos asociados a una litología más dura (grupos I y III)] siempre que no se consideren las lagunas asociadas en el análisis (Tabla 4). Esto viene justificado por el hecho de que las lagunas asociadas al Pozo de las Lomas, se encuentran a una altitud superior a la misma por lo que, además de presentar un tamaño menor, actúan a modo de trampa previa para las deposiciones (Fig. 3, PA1) y en el análisis computan como cuatro masas de agua, siendo su peso relativo elevado, por lo tanto al considerarlas se distorsiona la realidad observada.

Cabe destacar que si bien la relación profundidad-litología es estadísticamente significativa, la profundidad y dimensiones de las lagunas también dependen de las características concretas de su morfogénesis. Los lagos con mayores profundidades son el lago Ausente (LE3) y el pozo de Fuentes Carrionas (PA2) (Tabla 3), ambos con origen por circo glaciar y con potentes lenguas de hielo (Tabla 2). El circo del Ausente tuvo una lengua glaciar sobrealimentada por otras que descendían por la Sierra de Sentiles (Rodríguez Pérez, 1995), mientras que el circo de Fuentes Carrionas, debido a su altitud y orientación, superó el umbral de $2200 \mathrm{~m}$, descendiendo hacia el valle de Fuentes Carrionas (Pellitero Ondicol, 2012). Como masas de menor profundidad se han clasificado las temporales, Robledo (LE2), Chao (LE10) y Bustagil (LE11), todas ellas en estado avanzado de colmatación.

En cuanto a la hidrología, las lagunas presentan un origen epigénico, es decir, se distinguen corrientes superficiales que dan origen a las mismas (Figuras 3 y 4). No obstante, estos flujos en múltiples ocasiones son intermitentes debido a la presencia de canchales asociados a las

Tabla 4: ANOVA para las profundidades de las masas frente a los niveles de litología, dura (grupos I y III) y blanda (grupo II). Para el ANOVA: * $p$-valor $<0,05$, n.s.: no significativa. Table 4: ANOVA for depths against the lithological levels, hard (groups I and III) and soft (group II). For ANOVA: *p-valor $<0.05$, n.s. not significant.

\begin{tabular}{|c|c|c|c|}
\hline Variable & F & \multicolumn{1}{|c|}{ p-valor } \\
\hline \multicolumn{4}{|c|}{ Considerando asociadas } \\
\hline $\mathrm{Z}_{\max }$ & 3.73 & 0.068 & n.s. \\
\hline $\mathrm{Z}_{\operatorname{med}}$ & 2.94 & 0.102 & n.s. \\
\hline \multicolumn{4}{|c|}{ Sin considerar asociadas } \\
\hline $\mathrm{Z}_{\max }$ & 7.15 & 0.017 & $*$ \\
\hline $\mathrm{Z}_{\operatorname{med}}$ & 5.63 & 0.032 & $*$ \\
\hline
\end{tabular}

lagunas, por debajo de los cuales en ocasiones circulan las corrientes o regueros, desapareciendo en ciertos tramos (e.g. Malicioso). La conectividad aguas abajo de estas masas dependerá del hidroperiodo inter e intra anual y en muchos casos pudiendo llegar a ser endorreicas en la época estival (Tabla 3).

La morfología de las cuencas vertientes de cabecera, en su gran mayoría redondeadas (Tabla 5), favorecen una escorrentía distribuida en pequeñas y numerosas corrientes [e.g. LE5 (Figura 3)], que disminuyen en número cuando las morfologías son más alargadas y los valles en forma de U más marcados [e.g. LE10 (Figura 4)]. Esto denota el carácter complejo de estos sistemas y demuestra la necesidad de tratar estas masas de agua junto con sus cuencas a la hora de analizarlas y conservarlas.

Las curvas hipsométricas (proporción de superficie con relación a la altitud, es decir, superficie que existe por encima de una cota determinada) de las diferentes cuencas revelan perfiles propios de cuencas de cabecera (todas las lagunas se encuentran en las cabeceras de la red hidrológica), donde los tramos de mayor altitud presentan una escasa proporción superficial que aumenta a medida que se desciende (Figura 3). Este hecho, en términos individuales, supone mayores pendientes a medida que se asciende en altitud, y de la misma forma, se refleja al comparar los datos en conjunto donde las cuencas situadas a unas altitudes menores presentan pendientes menores (Tabla 5). Las curvas hipsométricas muestran los diferentes relieves y altimetrías de cada cuenca y permiten evaluar, en una primera fase, la vulnerabilidad de las masas de agua ante procesos erosivos. En este sentido, por ejemplo, en las cuencas de las lagunas PA1, LE2, LE3, LE5 y LE6, se puede observar una disminución en la pendiente antes de alcanzar las masas de agua que es susceptible de disminuir la velocidad de las corrientes descendentes favoreciendo la retención previa de sedimentos (Figura 5).

La diversidad muestral es coherente con las características litológicas y morfológicas observadas para las diferentes cuencas (Tablas 1, 2 y 5). Si bien, no se han detectado tendencias significativas entre los parámetros de cuenca y masa. Este hecho no es de extrañar dada la complejidad de estos sistemas y la cantidad de variables que intervienen en los procesos evolutivos de estas masas (Håkanson, 2005), siendo necesario destacar que probablemente las cuencas de los lagos y lagunas estudiados fueron mucho más amplias durante su génesis.

A pesar de que, en general, las lagunas de alta montaña presentan un aceptable estado de conservación debido a su marcado aislamiento (Fernández et al., 2000), son ecosistemas muy susceptibles a cambios en sus cuencas vertientes. En este sentido, el índice de área de la cuenca entre el volumen de la masa de agua $\left(\mathrm{S}_{\mathrm{C}} / \mathrm{V}_{\mathrm{L}}\right)$ da una idea de la sensibilidad y fragilidad de las unidades ante actuaciones antrópicas en sus respectivas cuencas, resultando elevado al compararlas con otras masas de agua [e.g. 0.32 para el Estany Redó en el Pirineo Central (Catalan, 1987) o 1.36 para el lago de Sanabria en Zamora (Vega et al., 2005)], lo que indica que, en general, los lagos y lagunas estudiados son frágiles ante actuaciones en sus cuencas vertientes. 


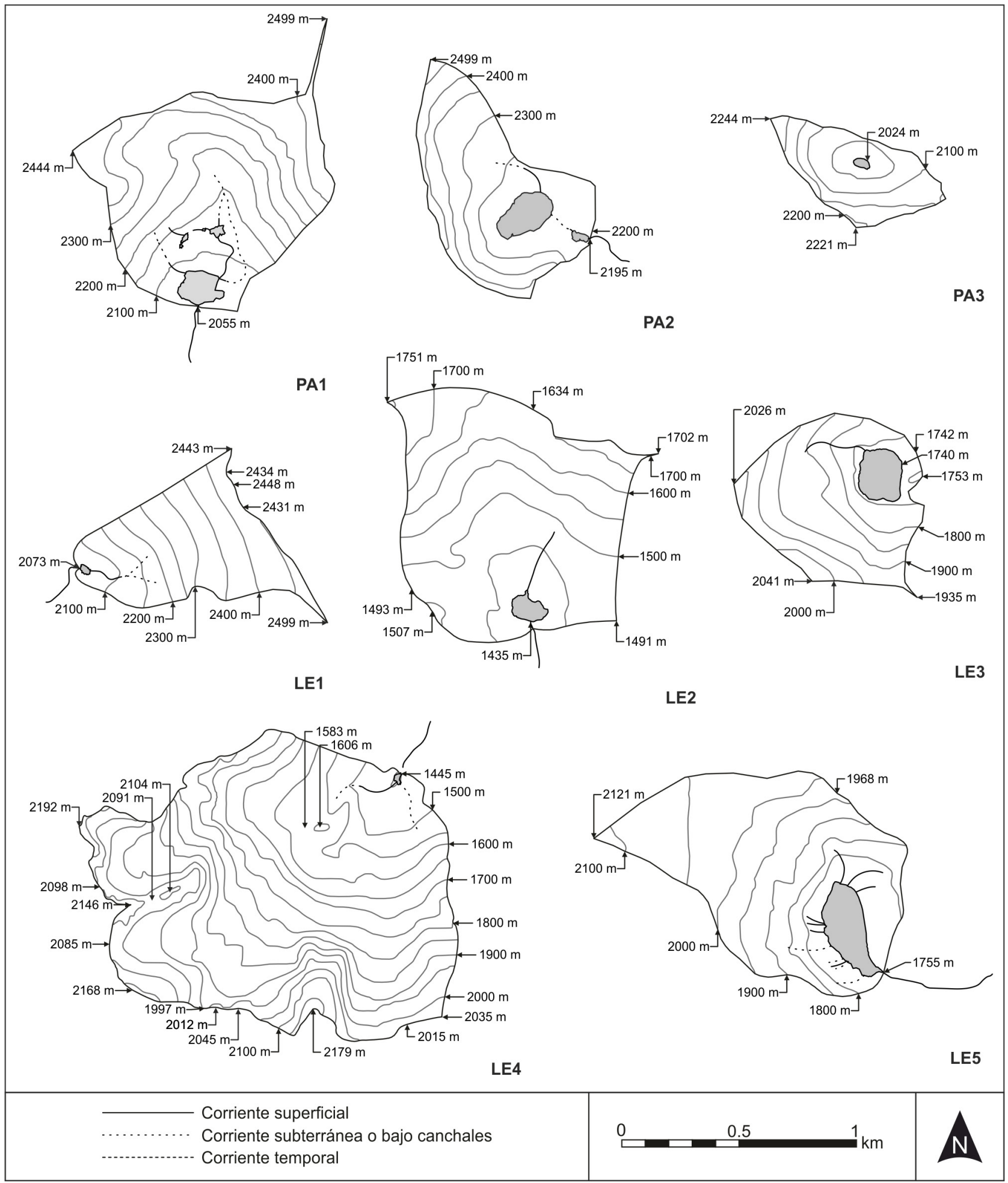

Figura 3: Topografía de las lagunas, definición de sus cuencas vertientes y funcionamiento hidrológico observado: PA1, PA2, PA3, LE1, LE2, LE3, LE4 y L5E.

Figure 3: Topography of lagoons and defining their catchments and hydrological performance: PA1, PA2, PA3, LE1, LE2, LE3, LE4 and LE5. 


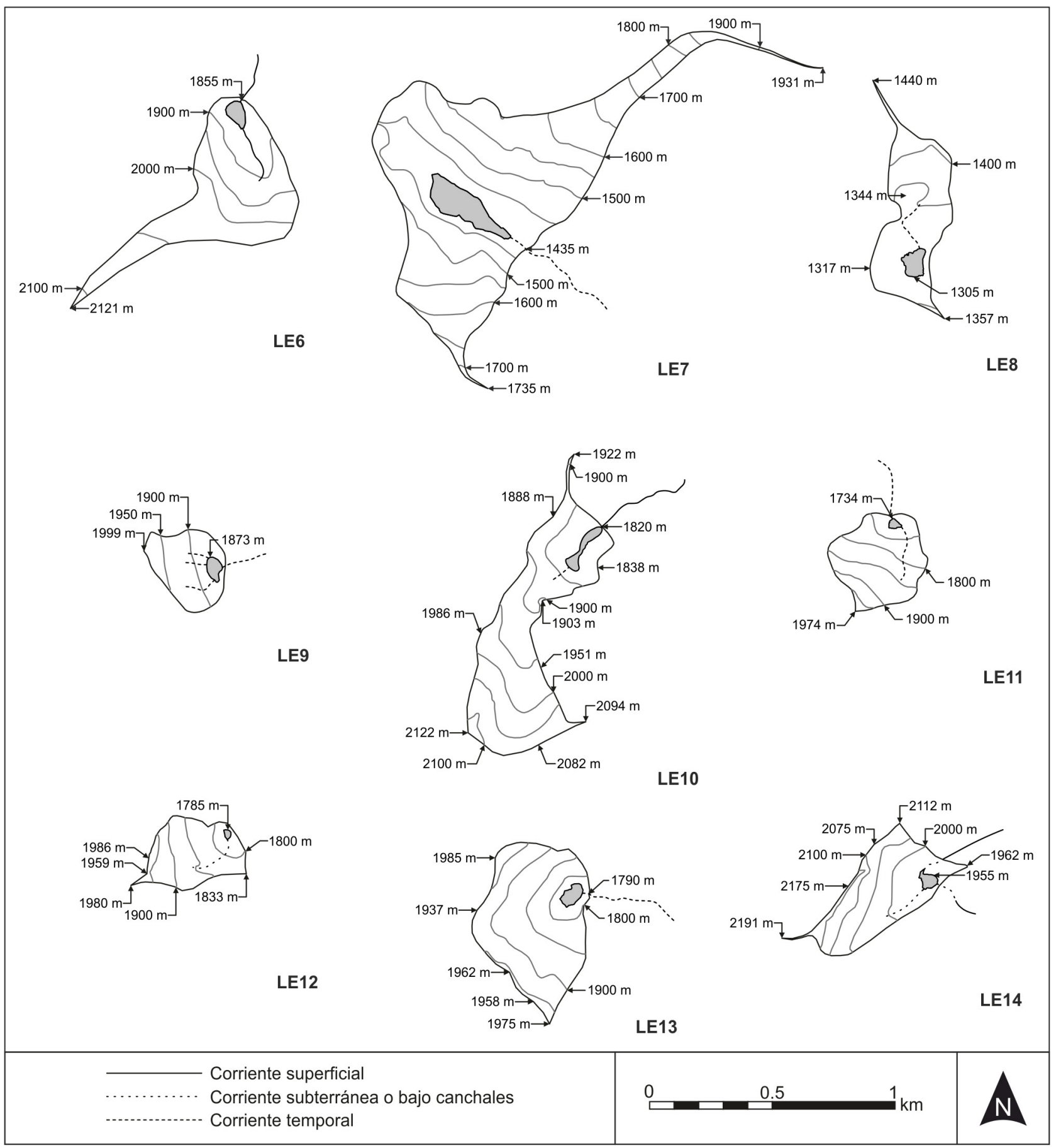

Figura 4: Topografía de las lagunas, definición de sus cuencas vertientes y funcionamiento hidrológico observado: LE6, LE7, LE8, LE9, LE10, LE11, LE12, LE13 y LE14 y definición de sus cuencas vertientes.

Figure 4: Topography of lagoons and defining their catchments and hydrological performance: LE6, LE7, LE8, LE9, LE10, LE11, LE12, LE13 and LE14. 


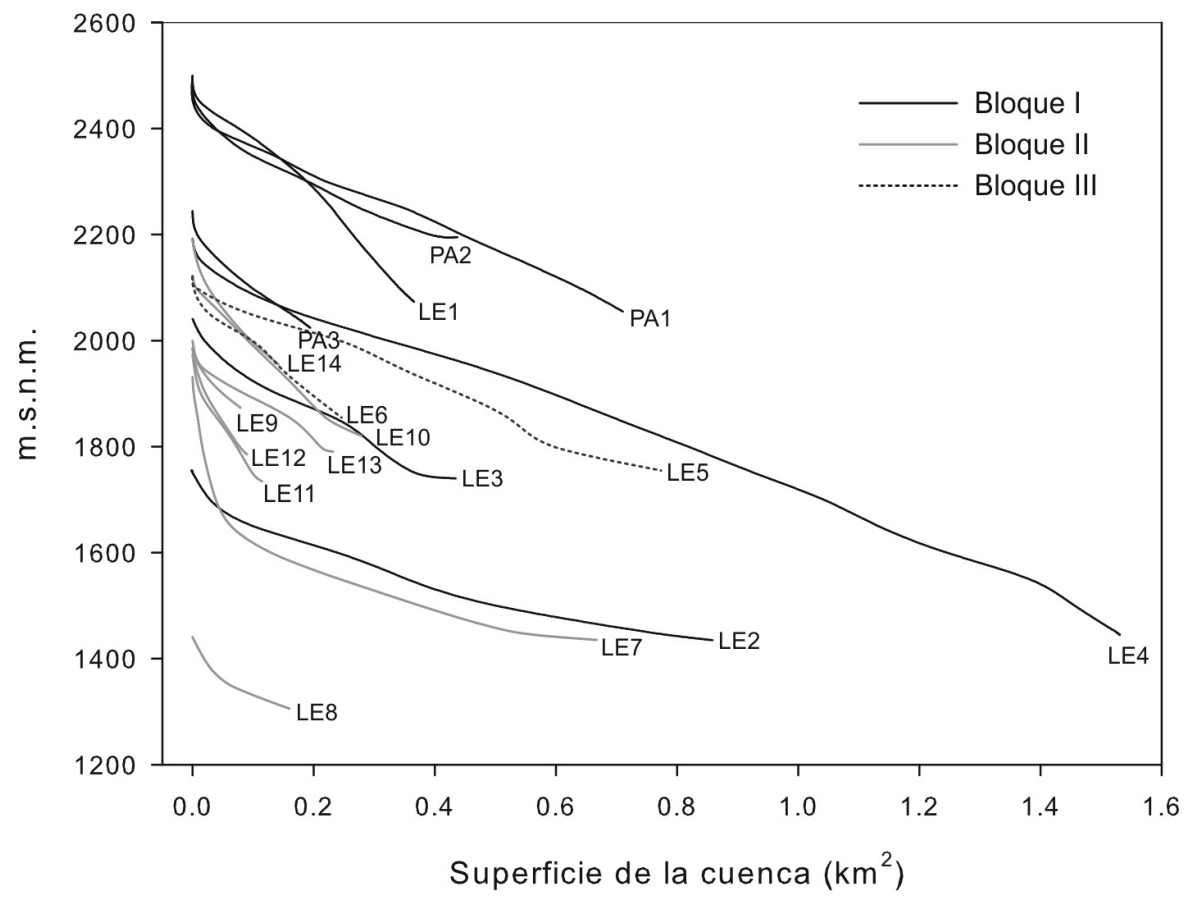

Figura 5: Curvas hipsométricas de las lagunas estudiadas.

Figure 5: Hypsometric curves of the studied lakes.

Tabla 5: Características físicas de las cuencas de las unidades lagunares estudiadas.

Table 5: Physical characteristics of basins of the studied lakes.

\begin{tabular}{|c|c|c|c|l|r|r|r|r|r|r|r|}
\hline Código & $\mathbf{S}_{\mathbf{C}}\left(\mathbf{m}^{\mathbf{2}}\right)$ & $\mathbf{P}_{\mathbf{C}}(\mathbf{m})$ & \multicolumn{2}{|c|}{ Tipología $\left(\mathbf{C}_{\mathbf{G}}\right)$} & $\mathbf{L}_{\mathbf{C}}(\mathbf{m})$ & $\mathbf{H}_{\max }(\mathbf{m})$ & $\mathbf{H}_{\min }(\mathbf{m})$ & $\mathbf{I}(\mathbf{\%})$ & $\mathbf{A}_{\operatorname{med}}(\mathbf{m})$ & $\mathbf{H}_{\mathbf{m e d}}(\mathbf{m})$ & $\mathbf{S}_{\mathbf{C}} / \mathbf{V}_{\mathbf{L}}$ \\
\hline PA1 & 710456.84 & 3953.80 & 1.31 & Ovalada & 1071.96 & 2499 & 2055 & 53.09 & 186 & 2241 & 5.82 \\
\hline PA2 & 437220.85 & 2840.77 & 1.20 & Redonda & 1006.58 & 2499 & 2195 & 59.35 & 96 & 2291 & 2.23 \\
\hline PA3 & 194475.86 & 1900.27 & 1.21 & Redonda & 820.07 & 2244 & 2024 & 67.97 & 83 & 2107 & 24.84 \\
\hline LE1 & 365849.99 & 3032.42 & 1.40 & Ovalada & 989.84 & 2499 & 2073 & 49.20 & 215 & 2288 & 618.47 \\
\hline LE2 & 858217.07 & 3870.07 & 1.17 & Redonda & 1139.87 & 1751 & 1435 & 35.99 & 107 & 1542 & - \\
\hline LE3 & 434546.52 & 2673.28 & 1.14 & Redonda & 817.47 & 2041 & 1740 & 56.27 & 119 & 1859 & 1.96 \\
\hline LE4 & 1526816.98 & 5268.09 & 1.19 & Redonda & 1475.53 & 2129 & 1445 & 81.20 & 370 & 1815 & 3340.52 \\
\hline LE5 & 773320.75 & 3644.75 & 1.16 & Redonda & 1352.93 & 2121 & 1755 & 38.46 & 166 & 1921 & 4.42 \\
\hline LE6 & 245865.41 & 2825.82 & 1.60 & Oblonga & 646.83 & 2121 & 1855 & 37.55 & 115 & 1970 & 7.59 \\
\hline LE7 & 667416.67 & 5719.49 & 1.96 & Fusiforme & 1073.47 & 1931 & 1435 & 44.21 & 100 & 1535 & 10.61 \\
\hline LE8 & 159936.81 & 2439.09 & 1.71 & Oblonga & 814.61 & 1440 & 1305 & 25.57 & 46 & 1351 & 41.01 \\
\hline LE9 & 79091.13 & 1074.52 & 1.07 & Redonda & 326.28 & 1999 & 1873 & 35.30 & 45 & 1918 & 36.30 \\
\hline LE10 & 278187.45 & 3299.37 & 1.75 & Fusiforme & 860.51 & 2122 & 1820 & 44.54 & 130 & 1950 & - \\
\hline LE11 & 114522.31 & 1306.52 & 1.08 & Redonda & 413.36 & 1974 & 1734 & 62.57 & 93 & 1827 & - \\
\hline LE12 & 89782.27 & 1307.09 & 1.22 & Redonda & 486.85 & 1980 & 1785 & 58.11 & 76 & 1861 & 221.55 \\
\hline LE13 & 232564.20 & 1944.40 & 1.13 & Redonda & 499.65 & 1985 & 1790 & 55.53 & 89 & 1879 & 10.13 \\
\hline LE14 & 146732.35 & 1967.35 & 1.44 & Ovalada & 6886.00 & 2191 & 1955 & 62.50 & 85 & 2040 & 84.63 \\
\hline
\end{tabular}




\section{Resumen y conclusiones}

El presente trabajo recoge y caracteriza morfológicamente, sin exclusión por tamaños, 17 complejos lagunares (22 lagos y lagunas) distribuidas en las provincias de Palencia y León. Su superficie hace que más del 50\% escape de las clasificaciones propuestas por la legislación estatal y europea. No obstante, dada su ubicación, a menudo remota y aislada, prácticamente todas ellas se encuentran bajo el amparo de alguna figura de protección.

El análisis estadístico realizado para las lagunas estudiadas señala una relación significativa entre la profundidad de las masas y su superficie, así como, de la profundidad de la masa y las litologías más resistentes. No obstante se destaca la influencia de las características concretas de su morfogénesis en relación a la morfología de la laguna y su cuenca.

En cuanto a la hidrología, la mayoría presenta un origen epigénico, diverso y complejo, menos evidente en la época estival, cuya conectividad aguas abajo dependerá de la magnitud y duración del hidroperiodo.

Las curvas hipsométricas reflejan el carácter de cabecera de las cuencas. Así mismo, a pesar de que los lagos y lagunas estudiados no parecen presentar ninguna relación significativa entre las variables de cuenca y de masa, su complejidad hidrológica y el elevado valor del cociente entre la superficie de las cuencas y el volumen de las masas de agua, para alguna de las unidades, refleja su fragilidad ante actuaciones e impactos en sus cuencas, haciendo necesario que cualquier medida encaminada a proteger estas lagunas tenga que aplicarse al conjunto de su cuenca vertiente.

\section{Agradecimientos}

La redacción de este estudio no hubiera sido posible sin el apoyo técnico del equipo del Grupo de Ecohidráulica Aplicada (GEA) de la Universidad de Valladolid (UVa). Así mismo, los autores desean agradecer al área de Ecología de la Universidad de León (ULe), por permitir la participación en el proyecto del Plan Nacional "Bases científicas para la definición del estado ecológico de lagos y lagunas de montañas de la cuenca del Duero", a la Dra. Felisa Santiago Ibarlucea (UVa) por su asesoramiento y a David Flores Leonor por su participación en los trabajos de campo. Así mismo, a los autores les gustaría agradecer las correcciones de los dos revisores anónimos que sin duda han ayudado a aumentar la calidad de este trabajo.

\section{Referencias}

Almodóvar, A. \& Elvira, B., 2000. Clasificación y conservación de los lagos de alta montaña de España según su ictiofauna. En: Granados, I. \& Toro, M. (eds.). Conservación de los lagos y humedales de alta montaña de la Península Ibérica. Servicio de Publicaciones. Universidad Autónoma de Madrid, 201-206 pp., Madrid.
Ampliación del catálogo de zonas húmedas de Castilla y León (aprobado por el Decreto 125/2001). 2001. Dirección General del Medio Natural. Junta de Castilla y León. Valladolid.

Arruebo, T., Pardo, A., Rodríguez, C., Lanaja, F.J. \& Del Va1le, J., 2009. Método específico para la evaluación medioambiental de los lagos de origen glaciar pirenaicos y su aplicación al lago de Sabocos. Pirineos, 164: 135-164. http:// dx.doi.org/10.3989/pirineos.2009.v164.33

Battarbee, R.W., 2005. Mountain lakes, pristine or polluted? Limnetica, 24 (1): 1-8.

Battarbee, R.W., Thompson, R., Catalan, J., Grytnes, J.A. \& Birks, H.J.B., 2002. Climate variability and ecosystem dynamics of remote alpine and arctic lakes: the MOLAR project. Journal of Paleolimnology, 28 (1): 1-6. http://dx.doi. org/10.1023/A:1020342316326

Carrillo, P., Delgado-Molina, J., Medina-Sánchez, J., Bullejos, F. \& Villar-Argaiz, M., 2008. Phosphorus inputs unmask negative effects of ultraviolet radiation on algae in a high mountain lake. Global Change Biology, 14 (2): 423-439. http://dx.doi.org/10.1111/j.1365-2486.2007.01496.x

Carrillo, P., Medina-Sánchez, J.M. \& Villar-Argaiz, M., 2002. The interaction of phytoplankton and bacteria in a high mountain lake: Importance of the spectral composition of solar radiation. Limnology and Oceanography, 47 (5): 12941306. http://dx.doi.org/ 10.4319/lo.2002.47.5.1294

Casamitjana, X., Colomer, J., Roget, E. \& Serra, T., 2006. Physical limnology in Lake Banyoles. Limnetica, 25 (1-2): 181-188.

Catalan, J., 1987. Limnologia de l'estany Redó (Pirineu Central). Tesis doctoral. Universidad de Barcelona.

Catalan, J., Camarero, L., Felip, M., Pla, S., Ventura, M., Buchaca, T., Bartumeus, F., de Mendoza, G., Miró, A. \& Casamayor, E.O., 2006. High mountain lakes: extreme habitats and witnesses of environmental changes. Limnetica, 25 (12): 551-584.

Catalan, J., Ventura, M., Brancelj, A., Granados, I., Thies, H., Nickus, U., Korhola, A., Lotter, A.F., Barbieri, A. \& Stuchlík, E., 2002. Seasonal ecosystem variability in remote mountain lakes: implications for detecting climatic signals in sediment records. Journal of Paleolimnology, 28 (1): 2546. http://dx.doi.org/10.1023/A:1020315817235

Catalan, J., Ballesteros, E., Camarero, L., Felip, M. \& Gacia, E., 1992. Limnology in the Pyrenean lakes. Limnetica, 8: 27-38.

Catálogo de zonas húmedas de Castilla y León (aprobado por el Decreto 194/1994). 1993. Consejería de Medio Ambiente y Ordenación del Territorio. Junta de Castilla y León. Valladolid.

Comisión Europea, 2000. Directiva 2000/60/CE del Parlamento Europeo y del Consejo de 23 de octubre de 2000 por la que se establece un marco comunitario de actuación en el ámbito de la política de aguas.

Cruz-Pizarro, L., Reche, I. \& Carrillo, P., 1994. Plankton dynamics in a high mountain lake (Las Yeguas, Sierra Nevada, Spain). Indirect evidence of ciliates as food source for zooplankton. Hydrobiologia, 274 (1): 29-35. http://dx.doi. org/10.1007/BF00014624

Fernández, P., García, J., Pérez, M.E., Pascual, M.L., Hidalgo, J., Rodríguez, A. \& Montes, C., 2000. La protección y conservación de los lagos y lagunas de alta montaña en la España peninsular. En: Granados, I. \& Toro, M. (eds.). Conservación de los lagos y humedales de alta montaña de la Península Ibérica. Servicio de Publicaciones. Universidad Autónoma de Madrid, 33-50 pp., Madrid.

Frochoso Sánchez, M. \& Castañón Älvarez, J.C., 1997. El relieve glaciar de la Cordillera Cantábrica. En: Gómez Ortiz, A. \& Pérez Alberti, A. (eds.). Las huellas glaciares de las montañas españolas. Universidad de Santiago de Compostela, 65-137, Santiago de Compostela. 
Fuentes-Pérez, J.F., 2009. Caracterización hidrogeomorfológica de lagos y lagunas de alta montaña en las provincias de Palencia y León (Castilla y León). ETSIIAA Palencia, Universidad de Valladolid (UVa).

Gallastegui, G., Heredia, N., Rodríguez Fernández, L. \& Cuesta, A., 1990. El stock de Peña Prieta en el contexto del magmatismo de la Unidad del Pisuerga-Carrión (Zona Cantábrica, N de España). Cuad. Lab. Xeol. Laxe, 15: 203-215.

García-Jurado, F., Guerrero, F., Galotti, A., Parra, G., Cruz-Pizarro, L., de Vicente, I., Rueda, F., Amores, V., Lucena, J., Rodríguez, V., León, P., Moreno-Ostos, B.B., Blanco, J.M., Zabala, L., Gilbert, J.D. \& Jiménez-Gómez, F., 2007. El Plancton de las lagunas de Sierra Nevada: necesidad de estudios transdisciplinares. $M+A$, revista electrónica de medioambiente, 4 (4): 1-10.

Håkanson, L., 2005. The importance of lake morphometry and catchment characteristics in limnology-ranking based on statistical analyses. Hydrobiologia, 541 (1): 117-137. http:// dx.doi.org/10.1007/s10750-004-5032-7

Huerga Rodríguez, A. (dir.), 1981. Mapa Geológico de España 1: 50.000, Hoja $\mathrm{n}^{\circ} 100$ y memoria explicativa. Instituto Geológico y Minero de España. Servicios de publicaciones del Ministerio de Industria y Energía. Madrid.

Leira, M., Bao, R. \& Vidal-Romaní, J.R., 1997. Evolución postglaciar de la laguna de Villaseca (NW de la Península Ibérica) a partir del análisis de diatomeas. Cuad. Lab. Xeol. Laxe, 22: 81-97.

Martínez de Azagra, A. \& Navarro, J., 1996. Hidrología forestal: el ciclo hidrológico, Servicios de Publicaciones de la Universidad de Valladolid, 286 pp., Valladolid.

Montes, C., 1995. Inventario y clasificación de lagos y humedales españoles. Tipificación y clasificación de humedales: bases para la elaboración de modelos de actuación y gestión prioritarios, DGOH-SEPOH-MOPTMA, Madrid.

Morales-Baquero, R., Carrillo, P., Cruz-Pizarro, L. \& SánchezCastillo, P., 1992. Southernmost high mountain lakes in Europe (Sierra Nevada) as reference sites for pollution and climate change monitoring. Limnetica, 8: 39-47.

Ordoñez, O.F., Flores, M.R., Dib, J.R., Paz, A. \& Farías, M.E., 2009. Extremophile culture collection from Andean lakes: extreme pristine environments that host a wide diversity of microorganisms with tolerance to UV radiation. Microbial Ecology, 58 (3): 461-473. http://dx.doi.org/10.1007/s00248009-9527-7

Pardo, L., 1948. Catálogo de los lagos de España, Ministerio de Agricultura.

Pascual, M.L., Rodríguez, A., Hidalgo, J., Borja, F., Díaz, F. \& Montes, C., 2000. Distribución y caracterización morfológica y morfométrica de los lagos y lagunas de alta montaña de la España peninsular. En: Granados, I. \& Toro, M. (eds.). Conservación de los lagos y humedales de alta montaña de la Península Ibérica. Servicio de Publicaciones. Universidad Autónoma de Madrid, 51-78, Madrid.

Pellitero Ondicol, R., 2012. Geomorfología, paleoambiente cuaternario y geodiversidad en el macizo de Fuentes $\mathrm{Ca}$ rrionas - Montaña Palentina. Tesis doctoral. Universidad de Valladolid.

Plan hidrológico de la parte española de la demarcación hidrográfica del Duero. Anejo 3: zonas protegidas. 2012. Minis- terio de Agricultura, Alimentación y Medio Ambiente. Madrid-Valladolid.

Rodríguez Fernández, L. R. (dir.), 1998. Mapa Geológico de España 1: 50.000, Hoja ${ }^{\circ} 102$ y memoria explicativa. Instituto Tecnológico GeoMinero de España. Servicios de publicaciones del Ministerio de Industria y Energía. Madrid.

Rodríguez Fernández, L. R. (dir.), 1990a. Mapa Geológico de España 1: 50.000, Hoja $\mathrm{n}^{\mathrm{o}} 79$ y memoria explicativa. Instituto Tecnológico GeoMinero de España. Servicios de publicaciones del Ministerio de Industria y Energía. Madrid.

Rodríguez Fernández, L. R. (dir.), 1990b. Mapa Geológico de España 1: 50.000, Hoja n ${ }^{\circ} 80$ y memoria explicativa. Instituto Tecnológico GeoMinero de España. Servicios de publicaciones del Ministerio de Industria y Energía. Madrid.

Rodríguez Fernández, L. R. (dir.), 1985. Mapa Geológico de España 1: 50.000, Hoja $\mathrm{n}^{\mathrm{o}} 106$ y memoria explicativa. Instituto Geológico y Minero de España. Servicios de publicaciones del Ministerio de Industria y Energía. Madrid.

Rodríguez Fernández, L. R. (dir.), 1982a. Mapa Geológico de España 1: 50.000, Hoja $n^{\circ} 101$ y memoria explicativa. Instituto Geológico y Minero de España. Servicios de publicaciones del Ministerio de Industria y Energía. Madrid.

Rodríguez Fernández, L. R. (dir.), 1982b. Mapa Geológico de España 1: 50.000 , Hoja $n^{\circ} 230$ y memoria explicativa. Instituto Geológico y Minero de España. Servicios de publicaciones del Ministerio de Industria y Energía. Madrid.

Rodríguez Fernández, L. R. (dir.), 1982c. Mapa Geológico de España 1: 50.000, Hoja $\mathrm{n}^{\circ} 77$ y memoria explicativa. Instituto Geológico y Minero de España. Servicios de publicaciones del Ministerio de Industria y Energía. Madrid.

Rodríguez Pérez, C., 1995. Estudio geomorfológico del Puerto de San Isidro. Ería, 36: 63-87.

Santos González, J., 2012. Glaciarismo y periglaciarismo en el Alto Sil, provincia de León (Cordillera Cantábrica), Universidad de León, León.

Santos González, J. \& Fernández Martínez, E., 2011. Guía de campo: patrimonio geológico en las reservas de la biosfera del Valle de Laciana y de Babia (León). En: FernándezMartínez, E. \& Castaño de Luis, R. (eds.). Avances y retos en la conservación del Patrimonio Geológico en España. Actas de la IX Reunión Nacional de la Comisión de Patrimonio Geológico (Sociedad Geológica de España). Universidad de León.

Skjelkvåle, B.L. \& Wright, R.F., 1998. Mountain lakes; sensitivity to acid deposition and global climate change. Ambio, 27 (4): 280-286.

Toro, M., Granados, I., Robles, S. \& Montes, C., 2006. High mountain lakes of the Central Range (Iberian Peninsula): Regional limnology and environmental changes. Limnetica, 25 (1-2): 217-252.

Toro, M. \& Granados, I., 2002. Restoration of a small high mountain lake after recent tourist impact: the importance of limnological monitoring and paleolimnology. Water, Air and Soil Pollution: Focus, 2 (2): 295-310. http://dx.doi. org/10.1023/A:1020127313830

Vega, J.C., De Hoyos, C., Aldasoro, J.J., De Miguel, J. \& Fraile, H., 2005. Nuevos datos morfométricos para el Lago de Sanabria. Limnetica, 24 (1-2): 115-122. 
ANEXO I

\begin{tabular}{|c|c|c|}
\hline \multicolumn{3}{|c|}{ Masa de agua: } \\
\hline \multicolumn{2}{|c|}{ Fecha: } & Región (DMA): Ibero macaronesica. \\
\hline \multicolumn{2}{|c|}{ Provincia: } & Cuenca: \\
\hline \multicolumn{2}{|l|}{ T.M.: } & Altitu (m.s.n.m.): \\
\hline \multicolumn{2}{|c|}{ Coordenadas UTM: } & $\mathrm{X}:$ \\
\hline Huso: & Zona: & Y: \\
\hline
\end{tabular}

- Red natura 2000:
$\square$ Zepa
$\square$ Lic
Zepa y Lic

- Caracterización del tipo genérico de la masa de agua:

Laguna: Pequeña extensión $<1$ ha y baja profundidad $(<2 \mathrm{~m})$, o una de las dos.

Lago: Extensión grande $>1$ ha y alta profundidad ( $>2 \mathrm{~m}$ ), o una de las dos.

- Origen hidrogeomorfológico:

\begin{tabular}{|c|c|c|c|}
\hline$\square$ Glaciar & $\square$ Periglaciar & $\square$ Gravitacional & $\square$ Fluvial \\
\hline Fólico & $\square$ Costero & $\square$ Disolución & $\square$ Tectónico \\
\hline Volcánico & $\square$ Meteorítico & $\square$ Biogénico & $\square$ Antrópico \\
\hline
\end{tabular}

- Origen hidrológico:
$\square$ Epigénico
Hipogénico
Mixto

- Si epigénico, aportaciones:

- Perímetro (m):

- Superficie (ha):
$\square<1$ ha
1 - 10 ha
10 - 50 ha
50 - 100 ha
$\square 100-1000$ ha
$1000-10.000$ ha
$\square>10.000$ ha

Observaciones: 


\section{ANEXO I (cont.)}

- Clasificación de Superficies (DMA) (km²):
$\square<0,5 \mathrm{~km}^{2}$
$\square 0,5-1 \mathrm{~km}^{2}$
$\square 0,5-1 \mathrm{~km}^{2}$
$10-100 \mathrm{~km}^{2}$
$\square>100 \mathrm{~km}^{2}$

- Tipología en función de la altitud (DMA):

$\square$ alto: $>800 \mathrm{~m} \quad \square$ altura media: 200 a $800 \mathrm{~m} \quad \square$ tierras bajas: $<200 \mathrm{~m}$

- Profundidad media $(\mathrm{m})$ :
$\square<1 \mathrm{~m}$
$\square 1-3 m$
$\square 3-5 \mathrm{~m}$
$5-10 m$
$\square 10-15 \mathrm{~m}$
$\square>15 \mathrm{~m}$

- Profundidad máxima (m):

- Tipología según la profundidad media (DMA) (m):
$\square<3 m$
$\square 3 \mathrm{~m}$ a $15 \mathrm{~m}$
$>15 \mathrm{~m}$

- Forma:

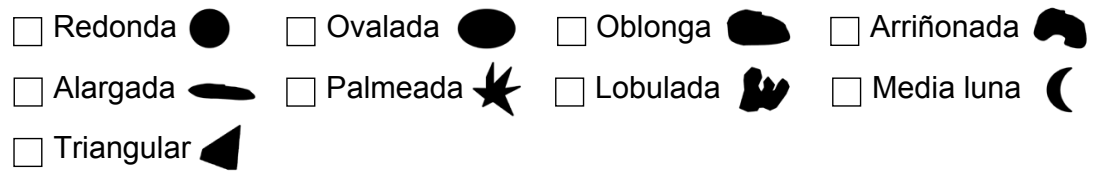

Observaciones:

- Estacionalidad:
$\square$ Permanente
Estacional

- Si estacional, tiempo de permanencia:

\section{- Conectividad:}

$\begin{array}{ll}\square \text { Exorreica } & \square \text { Endorreica } \quad \square \text { Endorreica parcial } \\ \square \text { Natural } & \square \text { Forzada (drenaje artificial) }\end{array}$




\section{ANEXO I (cont.)}

\section{- Si exorreica:}

Corriente que origina:

$\square$ Arroyuelo (anchura $<0,5 \mathrm{~m}$ y pendiente $<2 \%$ ) $\square$ Permanente $\square$ Estacional
$\square$ Arroyo (anchura $=0,5-5 \mathrm{~m}$ y pendiente $<2 \%$ ) $\square$ Permanente $\square$ Estacional
$\square$ Río (anchura $>5 \mathrm{~m}$ y pendiente $<2 \%$; en general permanente)
$\square$ Torrente (pendiente $>2 \%$ ) $\square$ Permanente $\square$ Estacional
$\square$ Otros lagos o lagunas
$\square$ Praderas húmedas
$\square$ Canales artificiales

- Naturaleza del lecho (si mixto estimar proporción):

$\square$ Limos (<0,75 mm) $\square$ Arenas $(0,75-2 \mathrm{~mm}) \quad \square$ Gravas (2 - 75 mm)
$\square$ Cantos (75 - 200 mm) $\square$ Bloques (200 - 500 mm) $\square$ Roca madre
$\square$ Bloques grandes y bolos (> $500 \mathrm{~mm})$

- Naturaleza de las laderas circundantes:

$\square$ Rocas duras (granitos, gneis, calizas, dolomías,...)

$\square$ Rocas blandas (Pizarras meteorizadas, margas, arcillas,...)

$\square$ Rocas y bolos

$\square$ Bolos y cantos (canchales)

Cantos y gravas

$\square$ Suelos (cultivos, pastos, bosques,...)

- Estructura de la zona y observaciones:

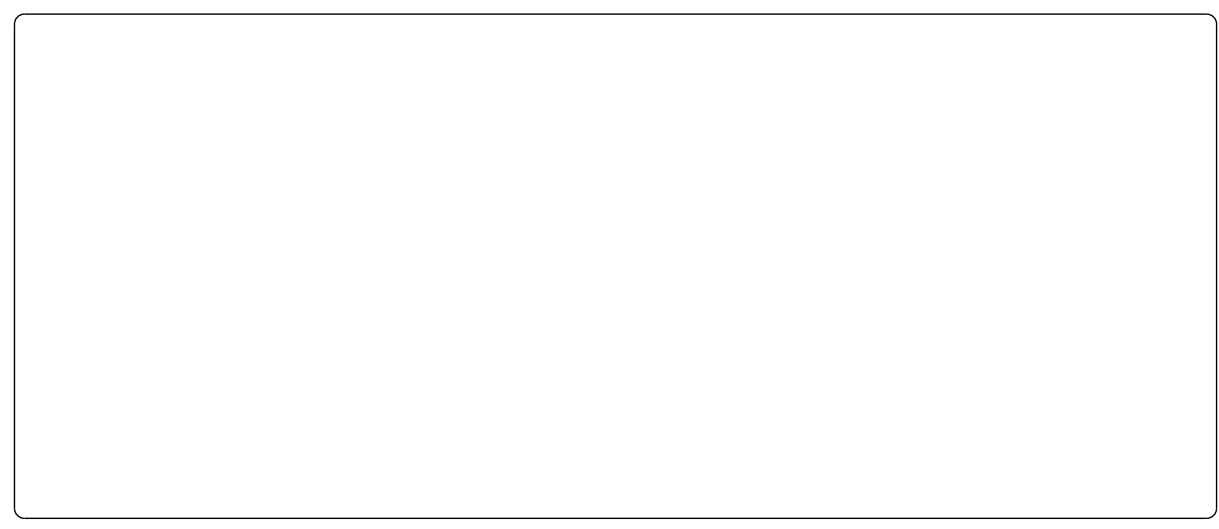

- Geología (DMA):

$\square$ Calcáreo $\quad \square$ Silíceo $\quad \square$ Orgánico




\section{ANEXO I (cont.)}

\section{- Erosión en las laderas circundantes:}

\section{Movimientos en masa:}

Rápidos:

$\square$ Desprendimientos $\square$ Deslizamientos $\square$ Coladas de barro Lentos:
$\square$ Solifluxión
Reptación

\section{Erosión superficial:}
$\square$ Laminar
$\square$ Regueros
Cárcavas

- Pendiente media de las laderas adyacentes:
$0-10 \%$
$\square 10-30 \%$
$\square 30-60 \%$
$60-100 \%$
$\square>100 \%$

- Transparencia del agua: (profundidad del disco de Secchi)
$\square<25 \mathrm{~cm}$
$\square 25-50 \mathrm{~cm}$
$\square 50-100 \mathrm{~cm}$
$1 \mathrm{~m}-1,50 \mathrm{~m}$
$\square 1,5 \mathrm{~m}-2 \mathrm{~m}$
$2 \mathrm{~m}-3 \mathrm{~m}$
$\square 3 \mathrm{~m}-5 \mathrm{~m}$
$\square>5 \mathrm{~m}$

- Alteraciones de forma:

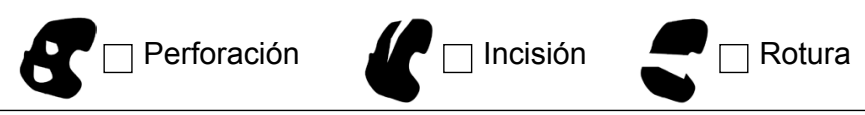

Observaciones:

- Calidad hidromorfologica (DMA):

Muy buen estado

$\square$ Buen estado

$\square$ Estado aceptable

$\square$ Mal estado 
18 • J. F. FUENTES-PÉREZ, J. NAVARRO HEVIA, J. RUIZ LEGAZPI, A. GARCÍA-VEGA

\section{ANEXO I (cont.)}

\section{- Presión antropogénica:}

$\square$ Instalaciones y actividades urbanas

$\square$ Industrial

$\square$ Agrícola

$\square$ Otras:

- Evaluación de presiones (impactos):

Crítico

$\square$ Severo

$\square$ Moderado

$\square$ Compatible

$\square$ Positivo

Observaciones: 